\title{
Possibilities of continual Measurement Alignment
}

\author{
Jakub Chromčák ${ }^{1 *}$ \\ ${ }^{1}$ Department of Geodesy, Faculty of Civil Engineering, University of Žilina, Slovakia
}

\begin{abstract}
The current legislative decree on Slovak republic is in the line with the applicable European Union standards and is based on the original legislative decrees supplemented and updated to meet the requirements of the European standards. The main European standard for track geometry is EN 13848. This standard specifies the requirements for aligning track geometry parameters that present the quality of track. Quality can be measured by different measuring devices mounted on tracking vehicles. This norm is applied to all track parameters. Each parameter is defined hand in hand with the requirements for its' measuring, methodology of processing and interpreting of the results. [1] That are the reasons, why there is not only needed to be focus of measurement technology, but on the processing and alignment as well. It is also necessary to focus to an inertial precision of the system. There are few problems, such as impossibility of having the identical detailed survey points on a rail superstructure.
\end{abstract}

\section{Introduction}

The issue of data processing has to be followed and the numerical solutions present as the best one. The mathematical solutions can be presented by application of linear and nonlinear regression, deformation analysis and the parameter estimation methods such as the least squares method, robust estimation method, maximum reliability method, collocation method for construction behaviours prediction and so. The result is mathematical definition of direction and altitude differences from the first basic stage or designed state. The necessity of each method application follows from the impossibility of having of identical points and the use of combined discrete and continual measurements. The priority during the processing is to preserve the basic parameters of data quality, precision of the measurement, and the reliability and objectivity of interpreted results.

\section{Regression analyses}

The regression analysis and correlation are used mainly in scientific and engineering analysis. Their main priority is to qualitative assessing of two or more dimensions expressed in a relationship written as:

\footnotetext{
* Corresponding author: jakub.chromcak@,fstav.uniza.sk
} 


$$
Y_{\mathrm{n}, 1}=X_{\mathrm{n}, \mathrm{k}} \beta_{\mathrm{k}, 1+} \varepsilon_{\mathrm{n}, 1},
$$

where vector $\mathrm{Y}$ represents the 1xn dimensioned vector of addicted observations (measurements), matrix $\mathrm{X}$ is known design matrix with dimension $\mathrm{nxk}, \beta$ is $\mathrm{kx} 1$ dimensioned vector of unknown parameters and $\varepsilon$ is $n x 1$ dimensioned vector of residues.[2]

The interesting quantities are statistically correlated, dependent. But there is an issue with not knowing of function type and the value of its' constants. The aim of regression analysis is additional definition of parameters, based on empirical, measured data.

One of possible ways is linear regression model, where the relation between the values has a linear form:

$$
y=A+B x .
$$

The best way to find out the values is using of basic linear model or Gauss-Marks model. The use of linear model is possible only in the straight parts of the track, there must be found other relationships between the values in the non-linear parts such as transition curves or arcs.

The interaction between the values in transition curve depends on curves' character, means the type of curve that was used. There is known a number of curves, like clothoid, cubic parabola, lemmiscate, curve of Bloss, cosinusoide etc. In these cases it is necessary to use non-linear relations and they cannot be processed by standard linear analysis. There are plenty of ways to process these values. First way is to try to linearize the non-linear relation (to find standard linear approximation of the non-linear function) and then use the linear regression. The other way is just to use least square method. Before using, it is necessary to have redundant measurements. The other ways is using of Gauss- Newton method, gradient method of Lederberg-Marquardt method. In the case of solving non-linear regression by using least square model are the final normal equations also non-linear. This result can seem like a problem in some cases and after-processing use. For use in processing of continual measurement, this type of regression can be used for expressing of the general equation of arc, written in parametrical way as:

$$
R^{2}=\left(x_{i}-X_{S}\right)^{2}+\left(y_{i}-Y_{S}\right)^{2},
$$

where the $\mathrm{R}$ is the radius of arc and $\left[\mathrm{X}_{\mathrm{S}}, \mathrm{Y}_{\mathrm{S}}\right]$ are the coordinates of the arc centre and $\left[\mathrm{x}_{\mathrm{i}}, \mathrm{y}_{\mathrm{i}}\right]$ are the coordinates of measured points placed on the arc.

This way of regression can be also used for transition curves. In Slovak Republic are regular to be used only cubic curve, curve of Bloss and for the railways using speed over $160 \mathrm{~km} / \mathrm{h}$ can be also used cosinusoide. Parametrical equation of cubic curve looks like:

$$
y_{\mathrm{i}}=\mathrm{ax}_{\mathrm{i}}^{3}+\mathrm{bx}_{\mathrm{i}}^{2}+\mathrm{cx}_{\mathrm{i}}+\mathrm{d},
$$

where the $a, b, c$ and $d$ are the parameters to be defined. Otherwise the equation of the curve of Bloss looks like:

$$
y_{i}=\frac{1}{R}\left(\frac{x_{i}^{4}}{4 L^{2}}-\frac{x_{i}^{5}}{10 L^{3}}\right)
$$

where $x_{i}$ is the length of the curve from its beginning in the tangent way, yi is the perpendicular length from the tangent in the same tangent distance from beginning, $R$ is the radius of curvature and $\mathrm{L}$ is the total length of the curve. 


\section{Analytic geometry methods}

The area of analytic geometry is the part of math, which is focused on studies of object geometrical shapes. The point of view on geometrical shapes is from its' analytical expression. Generally applies that if any point meets and satisfy the equation of object, than the point is the part of object. There is needed the coordination system to be Cartesian. If this condition is correct, than there is possible to write the equation of a line or a plane. There are many ways to define the line or the plane. The normal equation of the line is:

$$
\left(X-X_{0}\right) \cdot \vec{n}=0,
$$

where $\mathrm{X}_{0}$ is the point of the line and $\mathbf{n}$ is a normal vector of the line. By overwriting into coordinates we become the general equation of line:

$$
a x+b y+c=0,
$$

where $\mathrm{a}, \mathrm{b}$ and $\mathrm{c}$ are real numbers. There are two correct options, but only one regular. It is necessary to eliminate the $[0,0,0]$ incorrect option. [3] This two definitions of line are possible to be used only in $2 \mathrm{D}$ not in $3 \mathrm{D}$. The line can be also defined in $3 \mathrm{D}$ by parametrical definition:

$$
x=x_{0}+s_{1} t, \quad y=y_{0}+s_{2} t, \quad z=z_{0}+s_{3} t .
$$

These equations can be used to replace the determined parts of the track, also in transition curves or arcs. The correct interpretation of errors can be bit more difficult because of arc remoteness. In 3D space can be the shape of the track also replaced by the plane in vertical direction, the error of measurement can be interpreted as the distance of measured point from this plane. The general equation of plane looks like:

$$
a x+b y+c z+d=0 .
$$

The inaccuracy of point position can be interpreted as a distance of this point from the line/plane. The perpendicular distance of point from line defined by (7) is defined by equation:

$$
d=\frac{\left|a x_{0}+b y_{0}+c\right|}{\sqrt{a^{2}+b^{2}}} .
$$

If the line is defined by general equation, distance can be overwritten as:

$$
d=\sqrt{\frac{\left|\begin{array}{cc}
x_{0}-x & y_{0}-y \\
a & b
\end{array}\right|^{2}}{a^{2}+b^{2}}} .
$$


If the calculations needed the elevation to be included there is needed to find the perpendicular projection of the point into the $3 \mathrm{D}$ defined line. This projection can be interpreted as a point, which coordinates can be calculated from the equations:

$$
x_{Q}=\frac{b\left(b x_{0}-a y_{0}\right)-a c}{a^{2}+b^{2}} \quad y_{Q}=\frac{a\left(-b x_{0}+a y_{0}\right)-b c}{a^{2}+b^{2}} .
$$

The distance can be than easy calculated from coordinates of two points, where elevation of $Q$ point is given by interpolation. The distance than is:

$$
d_{3 D}=\sqrt{\left(x_{Q}-x\right)^{2}+\left(y_{Q}-y\right)^{2}+\left(z_{Q}-z\right)^{2}} .
$$

The other solution can also be the calculation of point-plane distance, the plane that replacing the line in $3 \mathrm{D}$ space. The shortest distance of point from the plane defined as general equation is:

$$
d_{3 D}=\frac{\left|a x_{0}+b y_{0}+c z_{0}+d\right|}{\sqrt{a^{2}+b^{2}+c^{2}}} .
$$

\section{Conclusions}

The current legislative decree on Slovak republic had changed. The change brought up new methods of measuring, by using continual measurements. But the changes also came into the calculation point of view. There is a number of ways, how to align the measurements and in the paper are shown some of them. There can be also used method of 2D or 3D transformation or other alignments' methods. Chosen methods are is some points simple and easy to be calculated. By using of analytic geometry, there rise the problems of error interpretation. Not in the straight parts but in the part of transition curves and the arcs. In analytic geometry they are replaced by the linear segments. The question to be answered is the length of these segments. If they were too long, the remoteness can be interpreted incorrectly as a mistake, if they were too short, the solution loose its' simplicity.

This article is the result of the implementation of the project VEGA 1/0275/17 "Application of numerical methods to define the changes of geometrical track position" supported by the Scientific Grant Agency of the Ministry of Education, science, research and sport of the Slovak Republic and the Slovak Academy of Sciences. This article is the result of the implementation of the project ITMS 26220220156 "Broker centre of air transport for transfer of technology and knowledge into transport and transport infrastructure" supported by the Research \& Development Operational Programme funded by the ERDF.

\section{References}

1. EN 13848-1 + A1 Railway applications - Track - Part 1: Characterisation of track geometry (2008)

2. Clarke R.B., The theory and application of Analysis of Variance, ISBN 978-0-47002566-6 (John Wiley \& Sons, New Jersey, USA, 2008)

3. Ižvoltová J., Cesnek T., Deformation Measurements of Ballast-less Railway Line, ISSN 2261-236X (XXVI R-S-P Seminar 2017) 
4. Ižvoltová J., Kot'ka V., Numerical adjustment of continual observations of ballast-less railway line, ISBN 978-619-7105-59-9 (SGEM 2016)

5. Šestáková J., Ižvoltová J., Mečár M., Monitoring pokusného úseku s pevnou jazdnou dráhou v oblasti tunela Turecký vrch, ISBN 978-80-554-1199-6 (STRAHOS 2016) 